\title{
sciendo
}

\section{On the Plausibility of Idealism: Refuting Criticisms}

\section{Bernardo Kastrup}

BIBLID [0873-626X (2017) 44; pp. 13-34]

\begin{abstract}
Several alternatives vie today for recognition as the most plausible ontology, from physicalism to panpsychism. By and large, these ontologies entail that physical structures circumscribe consciousness by bearing phenomenal properties within their physical boundaries. The ontology of idealism, on the other hand, entails that all physical structures are circumscribed by consciousness in that they exist solely as phenomenality in the first place. Unlike the other alternatives, however, idealism is often considered implausible today, particularly by analytic philosophers. A reason for this is the strong intuition that an objective world transcending phenomenality is a self-evident fact. Other arguments - such as the dependency of phenomenal experience on brain function, the evidence for the existence of the universe before the origin of conscious life, etc. - are also often cited. In this essay, I will argue that these objections against the plausibility of idealism are false. As such, this essay seeks to show that idealism is an entirely plausible ontology.
\end{abstract}

\section{Keywords}

Idealism, physicalism, panpsychism, cosmopsychism, mind-body problem.

\section{Introduction}

The mainstream physicalist ontology posits that reality is constituted by irreducible physical entities - which Strawson (2006: 9) has called 'ultimates' - outside and independent of phenomenality. According to physicalism, these ultimates, in and of themselves, do not instantiate phenomenal properties. In other words, there is nothing it is like to be an ultimate, phenomenality somehow emerging only at the level of complex arrangements of ultimates. As such, under 
physicalism phenomenality is not fundamental, but instead reducible to physical parameters of arrangements of ultimates.

What I will call 'microexperientialism', in turn, posits that there is already something it is like to be at least some ultimates (Strawson et al. 2006: 24-29), combinations of these experiencing ultimates somehow leading to more complex experience. As such, under microexperientialism phenomenality is seen as an irreducible aspect of at least some ultimates. The ontology of panexperientialism (Griffin 1998: 77-116, Rosenberg 2004: 91-103, Skrbina 2007: 21-22) is analogous to microexperientialism, except in that the former entails the stronger claim that all ultimates instantiate phenomenal properties.

Micropsychism (Strawson et al. 2006: 24-29) and panpsychism (Skrbina 2007: 15-22) are analogous - maybe even identical-to microexperientialism and panexperientialism, respectively, except perhaps in that some formulations of the former admit cognition-a more complex form of phenomenality - already at the level of ultimates, as an irreducible aspect of these ultimates.

While microexperientialism, panexperientialism, micropsychism and panpsychism entail that bottom-up combinations of simple subjects give rise to more complex ones, such as human beings, cosmopsychism (Nagasawa and Wager 2016) takes the opposite route. Indeed, "the first postulate of cosmopsychism is that the cosmos as a whole is the only ontological ultimate there is, and that it is conscious" (Shani 2015: 408, the emphasis is Shani's).

Finally, the ontology of idealism is characterized by a combination of two propositions: (a) phenomenal consciousness is irreducible; and (b) everything else - the whole of nature - is reducible to a unitary and universal phenomenal consciousness (henceforth, I shall refer to phenomenal consciousness simply as 'consciousness').

Idealism may be consistent with - even identical to - certain interpretations of cosmopsychism. According to Shani, for instance, cosmopsychism entails that "an omnipresent cosmic consciousness is the single ontological ultimate there is" (2015: 390). This perfectly embodies the defining tenet of idealism insofar as it implies that everything — including the physical — can be reduced to the phenomenal. Shani also writes that matter is the cosmos "in its appearance as exterior complement to the subjective realities of created selves" 
(2015: 412, emphasis added). The notion that matter is the phenomenal appearance of equally phenomenal dynamics is also eminently idealist. Therefore, these interpretations of cosmopsychism are essentially indistinguishable from idealism and I shall, henceforth, refer to them simply as idealism.

Other possible interpretations of cosmopsychism entail that the cosmos as a whole bears phenomenal properties - i.e. has inner life - but also has an aspect - the physical universe we can measurethat is irreducible to these phenomenal properties. Naturally, this implies a form of dual-aspect monism, a la Spinoza (Skrbina 2007: 88 ). Indeed, under these views the cosmos can still be said to be conscious, but not in consciousness. In the former case, the cosmos bears phenomenality; in the latter - which is the idealist view - the cosmos is constituted by phenomenality. Interpretations of cosmopsychism that are not consistent with idealism shall not be further addressed in this paper.

In what follows, I will attempt to rebut the most common objections to the plausibility of idealism. I will seek to show that these objections are based on circular reasoning, conflation, unexamined assumptions, and several other misconceptions.

\section{The felt concreteness objection}

English poet Samuel Johnson is said to have argued against Bishop Berkeley's idealism by kicking a large stone while exclaiming: 'I refute it thus!' (Boswell 1820: 218) Johnson was clearly appealing to the felt concreteness of the stone to suggest that it could not be just a figment of imagination. Indeed, the felt concreteness of the world is probably the main reason why people intuitively reject the notion that reality unfolds in consciousness. If a truck hits you, you will hurt, even if you are an idealist.

However, notice that appeals to concreteness, solidity, palpability and any other quality that we have come to associate with things outside consciousness are still appeals to phenomenality. After all, concreteness, solidity and palpability are qualities of experience. What else? A stone allegedly outside consciousness, in and by itself, is entirely abstract and has no qualities. If anything, by pointing to the felt concreteness of the stone Johnson was implicitly suggesting the 
primacy of experience over abstraction, which is eminently idealist.

We have come to automatically interpret the felt concreteness of the world as evidence that the world is outside consciousness. But this is an unexamined artifact of subliminal thought-models. Our only access to the world is through sense perception, which is itself phenomenal. The notion that there is a world outside and independent of the phenomenal is an explanatory model, not an empirical fact. No phenomenal quality can be construed as direct evidence for something outside phenomenality.

\section{The private minds objection}

As discussed in the Introduction, under idealism there is only one universal consciousness. Yet, at a personal level, our mental lives are clearly separate from one another. I do not have direct access to your thoughts and feelings and, presumably, neither do you to mine. Moreover, I do not seem to be aware of what is happening across the galaxy and, presumably, neither are you. So, if all reality is reducible to one universal consciousness, how can there be separate private minds such as yours and mine?

To make sense of this under idealism, we need to review a mental condition called dissociation (Braude 1995, Kelly et al. 2009: 167174 and 348-352, Schlumpf et al. 2014, Strasburger and Waldvogel 2015). Indeed, it is now well established in psychiatry that mental contents can undergo "a disruption of and/or discontinuity in [their] normal integration" (Black and Grant 2014: 191). This normal integration of mental contents takes place through chains of cognitive associations: a perception may evoke an abstract idea, which may trigger a memory, which may inspire a thought, etc. These associations are logical, in the sense that e.g. the memory inspires the thought because of a certain implicit logic linking the two. Integrated mentation can thus be modeled, for ease of visualization, as a connected directed graph. See Figure 1a. Each vertex in the graph represents a particular mental content and each edge a cognitive association logically linking mental contents together. Every mental content in the graph of Figure 1a can be reached from any other mental content through a chain of cognitive associations. Dissociation, in turn, can be visualized as what happens when the graph becomes disconnected, such 
as shown in Figure 1b. Some mental contents can then no longer be reached from others. Following the psychiatric convention, I shall refer to the subgraph with grey vertices as a (dissociated) alter.

a

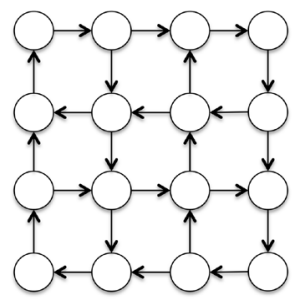

$b$

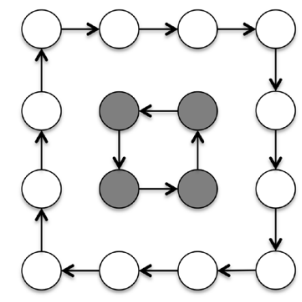

Figure 1. A connected graph (a) illustrating normal integration of mental contents, and a disconnected graph (b) illustrating dissociation and the corresponding formation of an alter (subgraph in grey).

Because cognitive associations are essentially logical, as opposed to spatio-temporal, the scheme of representation in Figure 1 allows for the simultaneous experience of multiple mental contents linked together in a connected subgraph. This is empirically justifiable: a perception, for instance, can be experienced at the same time as the thoughts it evokes and the emotions evoked by these thoughts. Moreover - and by the same token - the two disconnected subgraphs in Figure $1 \mathrm{~b}$ can also represent two concurrently conscious subjects of experience. The substantiation for this is again empirical: there is compelling evidence that different alters of the same psyche can be co-conscious (Kelly et al. 2009: 317-322, Braude 1995: 67-68).

An alter loses direct access to mental contents surrounding it, but remains integral to the underlying consciousness that constitutes it. The disconnection between an alter and surrounding mental contents is logical, not ontic. As an analogy, a database may contain entries that are not indexed and, therefore, cannot be reached, but this does not physically separate those entries from the rest of the database.

Dissociation can coherently explain how seemingly separate but concurrently conscious subjects of experience — such as you and me - can form under idealism: each is an alter of universal consciousness. And because each alter becomes unable to evoke the mental contents of another, their respective inner lives acquire a seemingly private character, even though they remain integral to the underlying 
consciousness that constitutes them.

\section{The stand-alone world objection}

If all there is is consciousness, does the world continue to exist when not consciously observed by a living being? A negative answer to this question seems extremely implausible yet difficult to avoid under idealism. Bishop Berkeley has famously attempted to circumvent it by appealing to a divinity, as captured in Ronald Knox's limerick, God in the Quad:

There was a young man who said 'God

Must find it exceedingly odd

To think that the tree

Should continue to be

When there's no one about in the quad.'

Reply:

'Dear Sir: Your astonishment's odd;

I am always about in the quad.

And that's why the tree

Will continue to be

Since observed by, Yours faithfully, God.'

Legitimate as an appeal to a divinity might have been in Berkeley's time, today more rigor is expected from a viable ontology. So how do we solve the problem of a stand-alone world under idealism?

With reference to the discussion in the preceding section, notice that, by definition, mental contents inside an alter of universal consciousness cannot directly evoke mental contents outside the alter, or vice-versa. But they can still influence or impinge on each other. Indeed, mental impingement across a dissociative boundary is empirically known. Lynch and Kilmartin (2013: 100), for instance, report that dissociated feelings can dramatically affect our thoughts, while Eagleman (2011: 20-54) shows that dissociated expectations routinely mold our perceptions. We can visualize this as in Figure 2a, wherein the partial overlap of adjacent vertices internal and external to the alter (cf. Figure 1b) represents mental impingement across its dissociative boundary. 
a

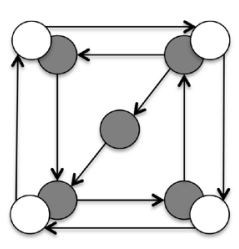

b

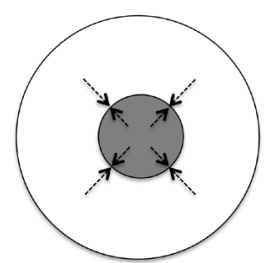

Figure 2. Mental contents impinging on the dissociative boundary of an alter, illustrated in two different ways (a) and (b).

Figure $2 \mathrm{~b}$ illustrates the exact same thing according to a simplified representation: the broader consciousness is represented as a white circle with an alter represented as a grey circle within it. The dashed arrows represent the impingement of external and internal mental contents on each other, across the alter's boundary. I will henceforth use this simplified representation.

Now notice that mental contents of universal consciousness that surround - but remain external to - an alter can impinge on the alter's boundary from the outside. Under idealism, it can be coherently argued that this is what gives rise to sense perceptions: the physical world around us is the extrinsic appearance on the screen of perception of phenomenality surrounding our respective alter. See Figure 3.

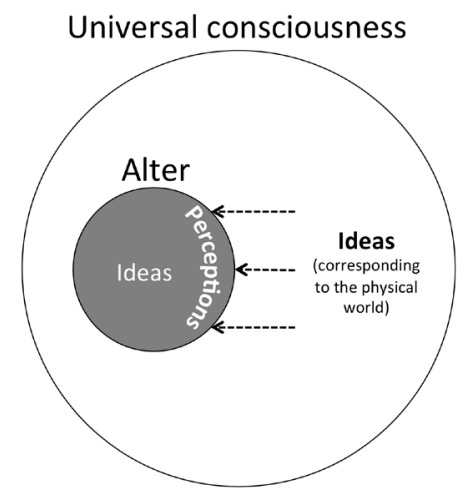

Figure 3. Mental contents of universal consciousness surrounding an alter can cause the alter's sense perceptions by impinging on its dissociative boundary. 
The stand-alone character of the world can thus be coherently explained: the physical world is a perceptual representation of phenomenality dissociated from our personal psyche and, as such, independent of our personal inner life. The physical world continues to exist — in the form of phenomenality outside our respective alter — even as we sleep.

\section{The autonomy of nature objection}

A closely related objection is this: nature unfolds according to patterns and regularities - the "laws of nature" - independent of our volition. Human beings cannot change these laws. But if nature is in consciousness, should that not be possible by a mere act of imagination?

This objection can be rebutted along the same lines as the previous one. However, there is a more direct and intuitive refutation. Notice that the implicit assumption here is that all mental activity is acquiescent to volition, which is patently false even in our own personal psyche. After all, by and large we cannot control our dreams, nightmares, emotions, and even many of our thoughts. They come, develop and go on their own terms. At a pathological level, schizophrenics cannot control their visions and people suffering from obsessive-compulsive disorder are constantly at the mercy of oppressive thoughts. There are numerous examples of conscious activity that escapes the control of volition. Often, we do not even recognize this activity as our own; i.e. we do not identify with it. It unfolds as autonomous, seemingly external phenomena, such as dreams and schizophrenic hallucinations. Yet, all this activity is unquestionably within consciousness. We perceive it as separate from ourselves because the part of our psyche that gives rise to this activity is dissociated from the ego, the part with which we do identify.

So that there is activity in universal consciousness that we do not identify with and cannot control is entirely consistent with idealism. This activity is simply dissociated from our ego and its sense of volition. 


\section{The shared world objection}

If all reality is in consciousness, then the world is akin to a dream. As such, idealism implies that we are all partaking in roughly the same dream. Yet, since our bodies are separate, we cannot be sharing a dream; or so the objection goes.

The objection begs the question by implicitly assuming that the body circumscribes dreaming consciousness, as opposed to the other way around. Only under this assumption does the impossibility of sharing a dream follow from the fact that bodies are separate. But under idealism, it is the body that is in universal consciousness, not consciousness in the body. Once this is properly understood according to the framework developed in the preceding sections, the rebuttal of this objection becomes rather straightforward: we all seem to inhabit the same world because our respective alters are surrounded by the same universal field of phenomenality, like whirlpools in a single stream. See Figure 4, which simply extends Figure 3 to multiple alters

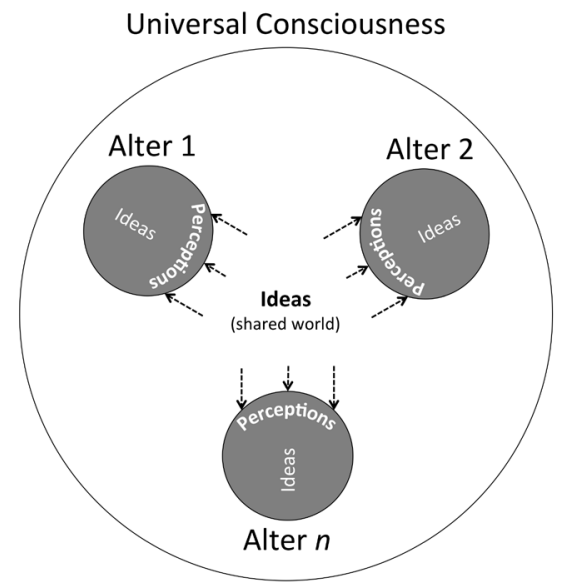

Figure 4. Alters of universal consciousness and their shared world. 


\section{The natural order objection}

The world we perceive around ourselves is governed by stable and orderly natural laws. Therefore, if the contents of perception are a representation of phenomenality in universal consciousness, then this phenomenality must be stable and orderly at root. But our own personal thoughts and emotions are notoriously unstable and disorderly. So how plausible is it that the order and stability we discern in the laws of nature represent thoughts or emotions in universal consciousness?

The misconception here, of course, is that of anthropomorphization: to attribute to universal consciousness as a whole cognitive characteristics known only in small dissociated aspects of it, such as human beings. Nothing in idealism precludes the possibility that phenomenality in universal consciousness unfolds according to very stable and orderly patterns and regularities, whose extrinsic appearance corresponds to the laws of nature. That our human thoughts and emotions seem rather reactive and unstable is a product of evolution under the pressures of natural selection within a particular planetary ecosystem. At a universal level, consciousness has not undergone such evolutionary pressures.

Under physicalism, the laws of nature are seen as irreducible causal patterns somehow built into the fabric of the cosmos. It is the dynamic unfolding of these patterns that leads to the order and complexity we see around ourselves. Under idealism, such irreducible causal patterns are posited to be somehow built into universal consciousness itself, instead of an objective fabric of space-time. Yet, beyond this distinction, they are the same patterns that physicalism entails, as inherent to consciousness as physical laws are allegedly inherent to the fabric of space-time. Idealism poses no extra difficulty than physicalism in this regard.

This can be better understood with a simple terminology move. Certain schools of psychology speak of 'psychological archetypes': innate, built-in templates according to which mental dynamics unfold (Jung 1991). As such, we can say that, under idealism, the laws of nature are the archetypes of universal consciousness. They are built-in templates according to which the "vibrations" of universal consciousness - i.e. phenomenality_develop, analogously to how 
the physical constraints of a vibrating surface determine its natural modes of vibration.

\section{The equivalence objection}

As we have seen in Sections 4 to 6, idealism acknowledges that there is a world outside personal psyches, since personal psyches are but dissociated aspects of a broader universal consciousness. The objection, then, is that the notion of a broad stream of phenomenality outside personal psyches is equivalent to the physicalist postulate of a world outside consciousness.

Except for solipsism, any viable ontology must entail at least one inference beyond direct experience. This is necessary to make sense of the fact that we all inhabit the same world beyond ourselves and are unable to change its governing laws. For this reason, physicalism infers the existence of a universe outside consciousness, which we all inhabit. Idealism, on the other hand, infers simply that consciousness itself extends beyond its face-value personal boundaries. This way, while physicalism postulates a fundamentally new ontological class next to experience, idealism simply extrapolates the boundaries of consciousness - the sole undeniable ontological class and primary datum of existence- - beyond those we can probe directly. To put it metaphorically, while idealism makes sense of reality by inferring that the Earth extends beyond the visible horizon, physicalism does so by inferring the existence of an isomorphic but ontologically distinct "shadow" Earth. Clearly, the former is a more parsimonious inference and, as such, not equivalent to the latter.

More importantly, the implications of idealism are radically different from those of physicalism. For instance, while physicalism implies that consciousness ends upon the death of the body, idealism implies merely the end of the corresponding dissociation, not of consciousness proper. I have elaborated on further differences in implications elsewhere (Kastrup 2015: 185-198).

\section{The primacy of brain function objection}

Not only are there (a) clear correlations between specific patterns of brain activity and reported inner experience (Koch 2004), we know 
that (b) physical interference with the brain — such as head trauma and the use of psychoactive drugs - can influence one's inner life rather dramatically. This may seem to suggest an arrow of causation pointing from a physical body outside consciousness to phenomenality, which would contradict idealism.

To make sense of observation (a), we need to briefly recapitulate earlier discussions. As we have seen in Section 3, under idealism private minds — such as our own human psyche — can be explained as dissociated alters of universal consciousness. We have also seen in Section 4 that the stand-alone world around us can be explained as the extrinsic appearance of phenomenality surrounding - but outside- our respective alter. Now, from the point of view of a given alter $A$, nothing prevents the dissociated mental activity of an alter $B$ from being part of the phenomenality surrounding $A$. $B$ is then part of $A$ 's world and, as such, must also have an extrinsic appearance on $A$ 's screen of perception. In other words, there must be something alters look like from a second-person point of view. And since we know from direct experience that our private inner life extends only to the boundaries of our metabolizing body - after all, we cannot perceive things that do not impinge on our skin or other sense organs, or move anything beyond our own body through direct intentionmetabolizing bodies seem prima facie to be the extrinsic appearance of dissociated alters of universal consciousness. If so, this means that all living beings have private inner lives in some way analogous to our own, but tables and chairs do not. The latter are simply part of the inanimate universe, which, as a whole, is the extrinsic appearance of phenomenality outside all alters.

Brain activity, of course, is integral to a metabolizing human body. Therefore, under idealism, brain activity is simply part of what one's private inner experiences - self-reflective and otherwise, as I will elaborate upon in the next section-look like from across a dissociative boundary. To put it another way, one's brain activity is part of a phenomenal representation of one's inner life. And of course, a representation must correlate with the phenomenal process it is the appearance of, without requiring anything ontologically distinct from consciousness. That this correlation is empirically observed is thus entirely consistent with idealism.

A possible counterargument here is this: the patterns of neural 
activity one can measure with functional brain scanners can be enormously complex in terms of information content; perhaps more complex than the contents of consciousness we have introspective access to. What does the extra complexity then correspond to? The key to answering this question is in the next section, wherein a distinction will be made between contents of consciousness we have introspective access to - i.e. can self-reflect upon - and contents of consciousness that, despite still being experienced, fall outside the reach of introspection. The extra complexity, insofar as it indeed is the case, corresponds to the latter.

Regarding observation (b) of the objection, the suggested arrow of causation is based on an unexamined but pervasive assumption: that the physical is in some sense distinct from, yet causally effective upon, the phenomenal. This is precisely what idealism denies. Under idealism, the physical is simply the contents of perception, a particular type of phenomenality. As such, what we call 'physical interference with the brain' is the extrinsic appearance of phenomenality external to an alter that disrupts the inner experiences of the alter from across its dissociative boundary. The disruption "pierces through" the boundary, so to speak. And that certain types of phenomenality disrupt other types of phenomenality is not only entailed by idealism, but also empirically trivial. After all, our thoughts disrupt our emotions - and vice-versa - every day. For the same reason that thoughts disrupt emotions, "physical interference with the brain" disrupts an organism's inner life. None of this contradicts idealism.

\section{The unconscious mentation objection}

In Libet's now famous experiments (1985), neuroscientists were able to record, a fraction of a second before subjects reported making a decision to act, mounting brain activity associated with the initiation of a simple voluntary action. At first sight, this would seem to indicate that decisions are made in a neural substrate outside consciousness, thereby contradicting idealism. I use Libet's experiments here merely as an example, for today we know of many other instances of seemingly unconscious mentation, such as moving one's foot halfway to the brake pedal before one becomes aware of danger ahead (Eagleman 2011: 5). Under idealism, since everything is in consciousness, 
there cannot be such a thing as unconscious mentation. So what is going on?

The misconception here is a conflation of consciousness proper with a particular configuration of consciousness. Indeed, to report an experience - such as making a decision to act or seeing danger ahead - to another or to oneself, one has to both (a) have the experience and (b) know that one has the experience, which Schooler (2002) called a 're-representation'. In other words, one can only report phenomenality that one is self-reflectively aware of at a metacognitive level. But self-reflection is just a particular configuration of consciousness, whereby consciousness turns in upon itself to experience knowledge of its own phenomenality (Kastrup 2014: 104-110). Nothing precludes the possibility that phenomenality takes place outside the field of self-reflection. In this case, we cannot report the phenomenality - not even to ourselves - because we do not know that we experience it.

The argument above is not idiosyncratic, for the existence of unreportable phenomenality is well established in neuroscience today (Tsuchiya et al. 2015, Vandenbroucke et al. 2014). Indeed, as elaborated upon by Schooler (2002), reportability is an extra function at a metacognitive level, on top of phenomenality proper. So the possibility that presents itself to us is that all mentation is actually conscious, even though we cannot report much of it. As such, the decisions made by Libet's subjects could well have been made in consciousness, but outside the field of self-reflection. The corresponding phenomenality then entered this field a fraction of a second later, thereby becoming reportable. Analogously, drivers may consciously see danger ahead before they can tell themselves that they see danger ahead. The appearance of unconscious mentation due to unreportability does not contradict idealism.

\section{The unconsciousness objection}

Along similar lines, the idea here is that, when we e.g. faint or undergo general anesthesia, we become seemingly unconscious. Yet, we do not cease to exist because of it, which may seem to contradict the idealist tenet that our body is the extrinsic appearance of conscious inner life. 
Let us consider this more carefully. Imagine that you wake up in the morning after hours of deep sleep. You may remember nothing of what happened during those preceding hours, concluding that you were unconscious all night. Then, later in the day, you suddenly remember that you actually had a very intense dream. So you were not unconscious all night, you simply could not remember your experiences.

Indeed, all we can assert with confidence upon coming round from episodes of seeming unconsciousness is that we cannot remember phenomenality occurring during those episodes. The actual $a b-$ sence of phenomenality is impossible to assert with confidence. As a matter of fact, many things we have traditionally associated with unconsciousness are now known to entail intense experiences. For instance, fainting caused by e.g. asphyxiation, strangulation or hyperventilation is known to correlate with euphoria, insights and visions (Neal 2008: 310-315, Rhinewine and Williams 2007, Retz 2007). G-force-induced loss of consciousness (G-LOC) is also known to correlate with "memorable dreams" (Whinnery and Whinnery 1990). There is even evidence for "implicit perception" during general anesthesia (Kihlstrom and Cork 2007).

Sleep, of course, is known to correlate with dreams. But even during phases of sleep wherein electroencephalogram readings show no dream-related neural activity, there are other types of activity that may correlate with non-recallable phenomenality distinct from dreams. Indeed, this is precisely what a recent study points out: "there are good empirical and theoretical reasons for saying that a range of different types of sleep experience, some of which are distinct from dreaming, can occur in all stages of sleep" (Windt, Nielsen, and Thompson 2016: 871, emphasis added). The authors identify three different categories of sleep experiences distinct from dreams: (a) non-immersive imagery and sleep thinking, (b) perceptions and bodily sensations, and (c) "selfless" states and contentless sleep experiences that may be similar to those reported by experienced meditators.

As such, what the empirical data shows is that episodes of seeming unconsciousness are associated with an impairment of memory formation or access, but not necessarily with absence of phenomenality. As a matter of fact, there are strong indications, as mentioned 
above, that the opposite is true.

\section{The solipsism objection}

Some conflate idealism with solipsism, the notion that the world is one's personal dream, all other living creatures being just figments of one's personal imagination. Under solipsism, there is nothing it is like to be other people; they have no inner life; they exist only as appearances in the personal psyche of the dreamer. As such, whatever empirical evidence one brings to bear and whatever one says to a solipsist must be regarded by the solipsist as figments of his or her own imagination, which renders solipsism unfalsifiable. So the objection here is that, by being unfalsifiable, solipsism - and therefore idealism - is beneath philosophical debate.

Naturally, idealism is not solipsism. Under idealism, there is something it is like to be other living creatures; they also have private inner lives. So idealists take other people seriously as legitimate sources of reported experiences and views, not just as figments of one's own imagination. Moreover, idealists acknowledge that there is a world outside and independent of their personal (dissociated) psyche, as discussed in Sections 4 to 6 . They simply do not acknowledge that this world is ontologically distinct from consciousness itself. Indeed, by acknowledging that dissociation in universal consciousness implies a world outside their own personal mentation, idealists look upon this world in a way entirely compatible with naturalism and scientific inquiry.

Unlike solipsism, idealism has the burden to explain observations non-trivially. Consider three basic facts that are often used to justify physicalism: (a) the laws of nature are independent of our personal volition; (b) we all seem to inhabit the same world; and (c) there are tight correlations between observable brain activity and reported inner life. Solipsism trivializes all three facts in lieu of actually making sense of them: the solipsist allegedly dreams them all up, rather arbitrarily. The idealist, on the other hand, by acknowledging the inner lives of other people and the autonomous nature of the world, has the burden to reconcile these three facts with the notion that reality unfolds in consciousness. If idealism is correct, (a) how come we cannot simply imagine a different and better world? If the world 
is akin to a dream in consciousness, (b) how come we are all having the same dream? If consciousness is not generated by the brain, (c) how come are there such tight correlations between brain activity and inner experience? These questions have already been answered in Sections 5, 6 and 9, respectively. The important point here is this: idealism is falsifiable in that, if it cannot answer these and other questions in terms of universal consciousness alone, it must be discarded.

\section{The cosmological history objection}

There is overwhelming evidence for the existence of the universe before conscious life arose. Therefore-or so the objection goesit is untenable to say that the universe exists in consciousness. This may strike some readers as obviously question-begging — which, of course, it is - but please bear with me for the sake of completeness.

The implicit assumption here is that consciousness arises only with biology, as a product of biology. Naturally, this is precisely what idealism denies. Under idealism, biology is merely the extrinsic appearance of dissociated, local differentiations of consciousness (i.e. alters), not the constituent or generator of consciousness. There was universal consciousness before such dissociated, local differentiations arose. And there was phenomenality in this universal consciousness corresponding to the inanimate universe prior to the origin of life.

\section{The implausibility of cosmic inner life objection}

The last objection I will address in this essay is, like the first, purely intuitive. It asks rhetorically: How plausible is it that the inanimate universe as a whole is the extrinsic appearance of some kind of universal inner life? The intuitive appeal of the question is understandable. After all, we only have introspective access to our own (dissociated) personal inner life, so to gauge the presence of other or broader inner life we depend on perceivable external indicators. In other people and animals, these indicators are their behavior. But within the extremely small range of space and time in which we live our lives - and even in which human history as a whole has unfolded -we simply cannot perceive any intuitively-appealing indicator of universal inner life. 
Yet, we can approach the question from a different angle. Consider a living brain exposed by surgeons during an operation. It is a very concrete object that can be seen, touched, cut, cauterized, etc. It is composed of the same types of atoms and force fields that make up the universe as a whole. There is nothing magical about a brain insofar as we can gauge in the screen of perception. And neither can we discern any intuitively-appealing indicator of inner life by simply looking at an exposed brain.

Nonetheless, we all know that "behind" the living brain lies the entire inner life of a person, with love affairs and heartbreaks, successes and disappointments, great adventures and quiet introspective insights, great joy and indescribable suffering. "Behind" that very concrete object under the surgeon's scalpel there lies a world of phenomenality. Counterintuitive or not, this is the way nature is: what we call physical structures - such as living brains — can correspond in some way to rich phenomenality. We may not know how this is so, but we do know that it is so.

Therefore, unless we solve the "hard problem of consciousness" (Chalmers 2003) and explain what makes brains different from the inanimate universe as a whole in this regard, if brains correspond to inner life it is not at all implausible that the inanimate universe as a whole could as well. After all, brains are made of the same "stuff" that the rest of the universe is also made of.

One could argue at this point that only particular structural and functional organizations of this "stuff," as found in brains, are conducive to the kind of information processing associated with human inner life. For instance, Tononi (2004) has shown that reportable experiences correlate only with complex networks of information integration in the brain. Although it has recently been shown that there are structural similarities between brains and the universe at its largest scales (Krioukov et al. 2012), it is implausible that analogous information integration takes place at a universal level. The distances and signal propagation times involved do not permit it (Siegel 2016).

However, the hypothesis offered here is not that the universe has human-like cognition and associated information integration. As a matter of fact, the hypothesis is not even that the universe has cognition, defined as the capacity to acquire knowledge or understanding. Instead, the claim is simply that there is raw experience- qualia, pure 
and simple - associated with the universe as a whole, which does not require anything like the kind of information integration underlying human self-reflection.

\section{Conclusions}

Idealism is a unique ontology in that, unlike physicalism and panpsychism, it asserts that physical structures are circumscribed by consciousness, as opposed to the other way around. Yet, analytic philosophy has traditionally considered idealism implausible. In this essay, I have argued that the alleged implausibility of idealism is based on misconceptions, such as:

- Unfounded intuition-e.g. taking the concreteness of the world to indicate its independence from consciousness, or asserting the implausibility of universal inner life;

- Lack of philosophical imagination-e.g. assuming that multiple private minds and a stand-alone world cannot be coherently reduced to a single universal consciousness;

- Demonstrably wrong assumptions - e.g. that all mental activity is acquiescent to volition;

- Question-begging - e.g. arguing that different people cannot share a dream because their bodies are separate, and arguing that the universe cannot be in consciousness because it existed before conscious life first arose;

- Anthropomorphization - e.g. taking all conceivable processes in consciousness to necessarily be unstable and disorderly;

- Failure to understand the implications of idealism - e.g. asserting that a field of phenomenality outside personal psyches is equivalent to a physical world outside phenomenality;

- Unexamined assumptions - e.g. that the physical is in some sense distinct from, yet causally effective upon, the phenomenal; 
- Conflation - e.g. conflating consciousness proper with selfreflection, conflating unconsciousness with failure to recall phenomenality, and conflating idealism with solipsism.

As such, idealism is an entirely plausible ontology that may offer the most parsimonious and explanatorily powerful option yet to make sense of reality. ${ }^{1}$

Bernardo Kastrup

Independent scholar Veldhoven, The Netherlands bernardo@bernardokastrup.com

\section{References}

Black, Donald and Grant, Jon. 2014. The Essential Companion to the Diagnostic and Statistical Manual of Mental Disorders. Fifth Edition. Washington: American Psychiatric Publishing.

Boswell, James. 1820. The Life of Samuel Johnson, LL. D., Volume 1. London: J. Davis, Military Chronicle and Military Classics Office.

Braude, Stephen. 1995. First Person Plural: Multiple Personality and the Philosophy of Mind. New York: Routledge.

Chalmers, David. 2003. Consciousness and its place in nature. In Blackwell Guide to the Philosophy of Mind, ed. by Stephen Stich and Ted Warfield. Malden: Blackwell.

Eagleman, David. 2011. Incognito: The Secret Lives of the Brain. New York: Canongate.

Griffin, David. 1998. Unsnarling the World-Knot. Eugene: Wipf \& Stock.

Jung, Carl. 1991. The Archetypes and the Collective Unconscious, ${ }^{\text {nd }}$ Edition. London: Routledge.

Kastrup, Bernardo. 2014. Why Materialism Is Baloney: How True Skeptics Know There Is No Death and Fathom Answers to life, the Universe, and Everything. Winchester: Iff Books.

Kastrup, Bernardo. 2015. Brief Peeks Beyond: Critical Essays on Metaphysics, Neuroscience, Free Will, Skepticism and Culture. Winchester: Iff Books.

Kelly, Edward et al. 2009. Irreducible Mind: Toward a Psychology for the $21^{1 s t}$ Century. Lanham: Rowman \& Littlefield.

Kihlstrom, John and Cork, Randall. 2007. Anesthesia. In The Blackwell Companion to Consciousness, ed. by Max Velmans and Susan Schneider.

${ }^{1}$ The author is grateful to an anonymous reviewer who has tirelessly taken it upon him/herself to help refine this paper as much as possible. This reviewer embodies the true spirit of peer-review as a constructive process. 
Oxford: Blackwell.

Koch, Christof. 2004. The Quest for Consciousness: A Neurobiological Approach. Englewood: Roberts \& Company Publishers.

Krioukov, Dmitri et al. 2012. Network Cosmology. Scientific Reports 2

Libet, Benjamin. 1985. Unconscious cerebral initiative and the role of conscious will in voluntary action. The Behavioral and Brain Sciences 8: 529-566.

Lynch, John and Kilmartin, Christopher. 2013. Overcoming Masculine Depression: The Pain Behind the Mask. New York: Routledge.

Nagasawa, Yujin and Wager, Khai. 2016. Panpsychism and priority cosmopsychism. In Panpsychism, ed. by Godehard Brüntrup and Ludwig Jaskolla. Oxford: Oxford University Press.

Neal, Richard. 2008. The Path to Addiction: And Other Troubles We Are Born to Know. Bloomington: AuthorHouse.

Retz. 2007. Tripping without drugs: experience with hyperventilation (ID 14651). Erowid.org: <www.erowid.org/exp/14651>

Rhinewine, Joseph and Williams, Oliver. 2007. Holotropic breathwork: the potential role of a prolonged, voluntary hyperventilation procedure as an adjunct to psychotherapy. The Journal of Alternative and Complementary Medicine 13: 771-776.

Rosenberg, Gregg. 2004. A Place for Consciousness. New York: Oxford University Press.

Schaffer, Jonathan. 2010. Monism: the priority of the whole. Philosophical Review 119: 31-76.

Schlumpf, Yolanda et al. 2014. Dissociative part-dependent resting-state activity in dissociative identity disorder: a controlled fMRI perfusion study. PloS ONE 9.

Schooler, Jonathan. 2002. Re-representing consciousness: dissociations between experience and meta-consciousness. Trends in Cognitive Science 6: 339-344.

Shani, Itay. 2015. Cosmopsychism: a holistic approach to the metaphysics of experience. Philosophical Papers 44: 389-437.

Siegel, Ethan. 2016. Ask Ethan: Is the universe itself alive? Forbes, 23 January: $<$ http://www.forbes.com/sites/startswithabang/2016/01/23/ask-ethan-isthe-universe-itself-alive>

Skrbina, David. 2007. Panpsychism in the West. Cambridge: MIT Press.

Strasburger, Hans and Waldvogel, Bruno. 2015. Sight and blindness in the same person: gating in the visual system. PsyCh Journal 4: 178-185.

Strawson, Galen et al. 2006. Consciousness and its Place in Nature. Exeter: Imprint Academic.

Tononi, Giulio. 2004. An information integration theory of consciousness. BMC Neuroscience 5: 42.

Tsuchiya, Naotsugu et al. 2015. No-report paradigms: extracting the true neural correlates of consciousness. Trends in Cognitive Science 19: 757-770.

Vandenbroucke, Annelinde et al. 2014. Seeing without knowing: neural signatures of perceptual inference in the absence of report. Journal of Cognitive Neuroscience 26: 955-969. 
Whinnery, James and Whinnery, Angela. 1990. Acceleration-induced loss of consciousness: a review of 500 episodes. Archives of Neurology 47: 764-776.

Windt, Jennifer, Nielsen, Tore, and Thompson, Evan. 2016. Does consciousness disappear in dreamless sleep? Trends in Cognitive Sciences 20: 871-882. 